\title{
Comparação de duas técnicas parasitológicas na detecção de enteroparasitos em catadores no sul do Brasil
}

\section{The comparison between two parasitological techniques for the detection of enteroparasites in waste pickers from the south of Brazil}

\author{
Juliana Carriconde Hernandes ${ }^{1}$, Érico Kunde Corrêa ${ }^{2}$, Luciara Bilhalva Corrêa ${ }^{3}$, Denise Gamio \\ Dias $^{4}$, Bruna Baccega ${ }^{5}$, Andréia Saggin Nagel ${ }^{6}$, Ana Lúcia Coelho Recuero ${ }^{7}$, Claudiomar Soares \\ Brod $^{8}$
}

\begin{abstract}
Resumo
Alguns pesquisadores e laboratórios clínicos utilizam apenas uma técnica para detecção de parasitos intestinais, podendo isto subestimar os achados. Enteroparasitos afetam principalmente populações com vulnerabilidade social, como os catadores. Alguns destes trabalhadores se reúnem em cooperativas, melhorando a condição de trabalho e o rendimento mensal, porém ainda não é o adequado, sendo por vezes insalubre. Nosso objetivo foi verificar a prevalência de enteroparasitos e comparar duas técnicas parasitológicas em suas medidas de efeito em catadores de material reciclável que trabalhavam em cooperativas no Sul do Brasil. Três amostras de fezes de 48 catadores, de seis cooperativas, foram coletadas em dias alternados, para a análise de parasitos. Duas técnicas de concentração de amostra foram utilizadas (Faust e Ritchie) e testadas para verificação da melhor a ser utilizada. A prevalência de parasitos em catadores foi de 62,5\% (30/48) utilizando as duas técnicas em paralelo, sendo que quando utilizadas sozinhas, essa prevalência foi menor. Diagnosticaram-se helmintos e protozoários, tanto patogênicos quanto comensais. A partir da análise estatística, provou-se que as técnicas parasitológicas fornecem melhores resultados quando utilizadas em paralelo, ou seja, quando utilizamos as duas técnicas juntas e em três amostras de fezes.
\end{abstract}

Palavras chave: Parasitos. Saúde pública. Reciclagem.

\begin{abstract}
Some researchers and clinical laboratories use just one technique for the detection of enteroparasites, allowing substandard findings. Enteroparasites mostly affect the socially vulnerable population, like the waste pickers. Some of these workers are gathered in cooperatives where work conditions and monthly wage are better. However, this environment is still not adequate being, sometimes, unsanitary. The objective was to study the enteroparasite prevalence and compare the two parasitological techniques in their effect measure in recyclable waste picker who were working in cooperatives in the south of Brazil. Three stool samples from 48 pickers from six cooperatives were collected in alternate days for parasite analysis. Two sample concentration techniques were used (Faust and Ritchie) and tested to verify which was the best to use. The parasite prevalence in waste picker was $62.5 \%(30 / 48)$, using the two techniques concurrently. When used individually, the prevalence was lower. Commensal and pathogenic helminths and protozoan were diagnosed. From the statistical analysis, it was proved that the
\end{abstract}

\footnotetext{
${ }^{1}$ Doutorado em Parasitologia pela Universidade Federal de Pelotas, Pelotas, Rio Grande do Sul, Brasil. E-mail: julianacarriconde@gmail.com

${ }^{2}$ Doutorado em Biotecnologia pela Universidade Federal de Pelotas, Pelotas, Rio Grande do Sul, Brasil. Professor adjunto do Centro de Engenharias, Universidade Federal de Pelotas, Pelotas, Rio Grande do Sul, Brasil.

${ }^{3}$ Doutorado em Educação Ambiental pela Universidade Federal do Rio Grande, Rio Grande, Rio Grande do Sul, Brasil. Professora adjunta do Centro de Engenharias, Universidade Federal de Pelotas, Pelotas, Rio Grande do Sul, Brasil.

${ }^{4}$ Doutorado em Educação pela Universidade Federal de Pelotas, Pelotas, Rio Grande do Sul, Brasil.

${ }^{5}$ Doutoranda em Parasitologia pela Universidade Federal de Pelotas, Pelotas, Rio Grande do Sul, Brasil.

${ }^{6}$ Mestre em Parasitologia pela Universidade Federal de Pelotas, Pelotas, Rio Grande do Sul, Brasil.

${ }^{7}$ Doutorado em Veterinária pela Universidade Federal de Pelotas, Pelotas, Rio Grande do Sul, Brasil.

${ }^{8}$ Doutorado em Biotecnologia pela Universidade Federal de Pelotas, Pelotas, Rio Grande do Sul, Brasil. Professor titular da Universidade Federal de Pelotas, Pelotas, Rio Grande do Sul, Brasil.
} 
parasitological techniques provide better results when used concomitantly, that is, when using the two techniques at the same time in three stool samples.

Keywords: Parasites. Public Health. Recycling.

\section{Introdução}

O exame parasitológico de fezes (EPF) por microscopia óptica ainda é muito utilizado para diagnóstico de enteroparasitos, mesmo este sendo um processo laborioso e necessitando de profissionais capacitados, pois permite resultados conclusivos no caso da positividade, além de baixos custos. ${ }^{(1)}$.

A técnica parasitológica utilizada como rotina na maioria dos laboratórios de análises clínicas consiste na técnica da sedimentação espontânea, sendo que este tipo de análise possui baixa sensibilidade, comprometendo assim os resultados dos exames e sendo mais bem empregada apenas em casos onde os indivíduos possuem infecções maciças. ${ }^{(2)}$ Por outro lado, técnicas parasitológicas que visam à concentração da amostra foram e estão sendo estudadas/empregadas com o intuito de aumentar a sensibilidade do diagnóstico e a probabilidade de detecção de ovos, cistos e oocistos de parasitos. ${ }^{(3)}$

As parasitoses intestinais, ainda constituem um problema de saúde pública para os países em desenvolvimento, sendo frequentemente diagnosticadas em população de baixa renda e de vulnerabilidade social, como no caso dos catadores de materiais reciclados. ${ }^{(4-5)}$ Estas infecções podem causar obstrução intestinal, diarréia e anemia, o que pode piorar a qualidade de vida e de trabalho destas pessoas. ${ }^{(6)}$

Os trabalhadores que manuseiam os materiais recicláveis enfrentam problemas como a exclusão social e a hostilidade, sendo muitas vezes vistos com desprezo, além da insalubridade a qual estão expostos, ${ }^{(7)}$ pois a atividade de triagem dos resíduos está predisposta a riscos como biológico, físico, químico, ergonômico e psicossocial, entre outros. ${ }^{(8)}$

O ambiente das cooperativas ainda não é o adequado, principalmente pelo contato direto do trabalhador com os resíduos que não sofreram correta segregação na fonte e foram destinados inadequadamente, tais como: resíduos de serviço de saúde, fraldas descartáveis, papel higiênico, alimentos, absorvente íntimo, pilhas e lâmpadas. ${ }^{(9)}$ Algumas cooperativas possuem animais domésticos circulando no seu entorno e mesmo dentro delas, sendo que estas condições atreladas ao não uso de equipamentos de proteção individual (EPI), geram um ambiente propício para a transmissão de agentes de doenças.

Diante do exposto da importância da utilização de técnicas parasitológicas apropriadas, bem como a situação dos catadores de materiais recicláveis, o presente trabalho teve como objetivo verificar a prevalência de enteroparasitos e comparar duas técnicas parasitológicas em suas medidas de efeito em catadores de material reciclável que trabalhavam em cooperativas no Sul do Rio Grande do Sul, Brasil.

\section{Material e Método}

A pesquisa foi realizada entre maio de 2016 a dezembro de 2016, com 48 trabalhadores pertencentes a seis cooperativas de triagem de material reciclável em um município de 350 mil habitantes, no Sul do Brasil.

Para aumentar a capacidade de detectar espécies de parasitos eliminadas nas fezes, utilizaram-se os métodos chamados processos de enriquecimento, ou técnicas de diagnóstico em paralelo (dois ou mais testes são aplicados ao mesmo indivíduo e só aqueles com resultados negativos em todos os testes são considerados livres de parasito). Este procedimento é útil quando um único teste não consegue detectar indivíduos em momentos distintos da parasitose.

Foram coletadas três amostras fecais de cada trabalhador, colhidas em dias alternados. Foi explicado o procedimento de coleta do material e entregue o primeiro frasco coletor contendo líquido conservante MIF (Merthiolato, Iodo, Formol). O segundo frasco era entregue quando se recolhia a primeira amostra, e o terceiro frasco quando se recolhia a segunda amostra. A análise parasitológica foi realizada através das técnicas de concentração de Faust ${ }^{(10)}$ que é um método de centrifugo-flutuação, para a detecção de cistos e oocistos de protozoários e ovos leves de helmintos; e de Ritchie $^{(11)}$ que é um método de centrífugosedimentação, utilizado para a detecção de larvas e ovos mais pesados de helmintos, além de cistos de protozoários.

Um questionário semiestruturado foi aplicado para a obtenção de dados de identificação de cada 
cooperado, utilização de EPI's, presença de animais em casa e na cooperativa, consumo de carne crua ou malcozida, além de uma observação geral do ambiente, para posterior cruzamento de dados.

A leitura das lâminas foi realizada através de microscópio óptico, com aumento de 100x e 400x.

Os resultados foram submetidos à análise estatística, utilizando o programa Epi-Info versão 6.04, efetuandose o teste do Qui-quadrado $(\chi 2)$. Os resultados foram considerados estatisticamente significativos quando observado $\mathrm{p}<0,05$; bem como a avaliação do intervalo de confiança, onde este não deveria incluir a unidade ${ }^{(1)}$. Foi ainda determinado o valor do risco relativo (RR) com intervalo de confiança (CI) de 95\% e medidas de efeito dos testes como, Sensibilidade (S), Especificidade (E), Valor Preditivo Positivo (VPP), Valor Preditivo Negativo (VPN) e Coeficiente de Correlação Kappa (ССҚ) para comparação dos resultados dos métodos de diagnóstico.

O ССҚ avalia o quão associadas estão duas ou mais observações e suas medidas variam de fraca $(<0,20)$; regular $(0,21$ a 0,40$)$; moderada $(0,41$ a 0,60$)$; substancial $(0,61$ a 0,80$)$; e excelente $(0,81$ a 1,00$)$. $\mathrm{O}$ que se deseja quando se trabalha com técnicas em paralelo é que não haja esta correlação excelente, pois assim não haveria a necessidade das duas técnicas, uma vez que uma traduziria praticamente o resultado de outra.

A pesquisa foi aprovada pelo comitê de ética da Faculdade de Medicina da Universidade Federal de Pelotas (RS), sob o n ${ }^{\circ}$ 16762213.9.0000.5317.

\section{Resultados}

\section{Análise parasitológica}

A menor prevalência para enteroparasitos ocorreu na terceira amostra, onde com Ritchie encontrou-se $18,8 \%$ e com Faust 25,0\%, enquanto que na análise em paralelo a prevalência pelas técnicas de Faust e/ ou Ritchie foi de 27,1\%. A análise das duas primeiras amostras de fezes, isoladamente, revelou prevalências idênticas (20 catadores), entretanto os indivíduos parasitados não eram todos os mesmos. Nas prevalências individuais para Ritchie1 ou Ritchie2, encontrou-se 29,2\% e para Faust1 ou Faust2, 33,3\%. $\mathrm{Na}$ análise em paralelo verificou-se uma prevalência de 41,7\%. Quando avaliamos as duas em conjunto, Ritchie12 e/ou Faust12, a prevalência sobe para $56,3 \%$. Na análise em paralelo da prevalência final,
Faust123 foi de 52,1\%, e Ritchie123 foi de 43,8\%. Considerando o resultado das duas técnicas em paralelo, a prevalência aumentou para $62,5 \%$ (Tabela 1).

Quanto ao Қ (Tabela 1), verifica-se que o maior número de parasitados foi nos resultados de Ritchie123 x Faust123, com um valor de Қ moderado, com desvio à esquerda $(0,419689)$. O maior valor de $\mathrm{K}$ encontrado $(0,696970)$, correlação substancial, foi verificado na análise da terceira amostra de fezes, onde se encontrou 13 parasitados, o menor valor achado.

Todas as análises estatísticas dos diferentes cruzamentos de técnicas e amostras de fezes apresentaram resultados significativos $(p<0,05)$, como se pode observar no RR, que mediu quantas vezes mais prováveis foram os trabalhadores positivos ao Ritchie de serem também positivos no Faust, do que os catadores negativos ao Ritchie. O menor RR foi de 2,29 e o maior de 8,67 (Tabela 1).

\section{Parasitados}

Como trabalhamos com três amostras de fezes sequenciais, podemos avaliar as medidas de efeito entre as técnicas de Faust e Ritchie, bem como o valor de Қ (Tabela 2).

Com relação aos parasitados (30 catadores), Faust detectou $83,3 \%(25 / 30)$ de indivíduos parasitados e Ritchie detectou 70,0\% (21/30) (Tabela 2.1). A comparação de Ritchie em relação à Faust e as medidas de efeito (S, E, VPP, VPN e K) para parasitados, protozoários e helmintos encontram-se nas Tabelas 2.1 à 2.3 .

A presença de protozoários foi verificada em 15 catadores, onde Faust detectou 93,3\% (14/15) dos indivíduos enquanto Ritchie detectou 40,0\% (6/15) (Tabela 2.2). Quanto a Helmintos, verificou-se a presença em 24 catadores, onde Faust detectou maior número de parasitados $(75,0 \%$ - 18/24) enquanto que Ritchie detectou 66,67\% (16/24) (Tabela 2.3).

O monoparasitismo ocorreu em 17 catadores, dos quais $70,59 \%$ (12/17) foram detectados por Faust e $52,94 \%$ (9/17) por Ritchie. Comparando Ritchie em relação à Faust nos monoparasitados, as medidas de efeito para protozoários e para helmintos, encontramse nas Tabelas 2.4 à 2.6.

O poliparasitismo ocorreu em 13 catadores de materiais recicláveis, onde $100,0 \%$ 13/13) foram detectados por Faust e $92,31 \%$ (12/13) por Ritchie. Comparando Ritchie em relação à Faust e aos 
Tabela 1 - Medidas de efeito, Risco Relativo, Qui quadrado entre os testes de Faust (F) e Ritchie (R) na detecção de enteroparasitos em 48 catadores de material reciclável das seis cooperativas do Município de Pelotas (RS) entre maio e dezembro de 2016.

\begin{tabular}{|c|c|c|c|c|c|c|c|c|c|c|c|c|c|c|c|c|c|c|c|}
\hline \multirow[t]{2}{*}{ Ritchie $_{1}$} & \multicolumn{2}{|c|}{ Faust $_{1}$} & \multirow[t]{2}{*}{ Total } & \multirow[t]{2}{*}{ Ritchie $_{2}$} & \multicolumn{2}{|c|}{ Faust $_{2}$} & \multirow[t]{2}{*}{ Total } & \multirow[t]{2}{*}{ Ritchie $_{3}$} & \multicolumn{2}{|l|}{ Faust $_{3}$} & \multirow[t]{2}{*}{ Total } & Ritchie $_{12}$ & \multicolumn{2}{|c|}{ Faust $_{12}$} & \multirow[t]{2}{*}{ Total } & \multirow[t]{2}{*}{ Ritchie $_{123}$} & \multicolumn{2}{|c|}{ Faust $_{123}$} & \multirow[t]{2}{*}{ Total } \\
\hline & Pos 1 & Neg. & & & Pos & Neg. & & & Pos & Neg & & & Pos & Neg & & & Pos & Neg & \\
\hline Pos & 10 & 4 & 14 & Pos & 10 & 4 & 14 & Pos. & 8 & 1 & 9 & Pos. & 15 & 5 & 20 & Pos. & 16 & 5 & 21 \\
\hline $\mathrm{Neg}$ & 6 & 28 & 34 & Neg & 6 & 28 & 34 & Neg. & 4 & 35 & 39 & Neg. & 7 & 21 & 28 & Neg. & 9 & 18 & 27 \\
\hline Total & 16 & 32 & 48 & Total & 16 & 32 & 48 & Total & 12 & 36 & 48 & Total & 22 & 26 & 48 & Total & 25 & 23 & 48 \\
\hline I.C. $95 \%$ & $\%$ & $\mathbf{L}_{\mathrm{i}}$ & $\mathbf{L}_{\mathrm{s}}$ & I.C. $95 \%$ & $\%$ & $\mathbf{L}_{\mathrm{i}}$ & $\mathbf{L}_{\mathrm{s}}$ & I.C. $95 \%$ & $\%$ & $\overline{\mathbf{L}_{\mathrm{i}}}$ & $\mathbf{L}_{\mathrm{s}}$ & I.C. $95 \%$ & $\%$ & $\mathbf{L}_{\mathrm{i}}$ & $\mathbf{L}_{\mathrm{s}}$ & I.C. $95 \%$ & $\%$ & $\mathbf{L}_{\mathrm{i}}$ & $\mathbf{L}_{\mathrm{s}}$ \\
\hline Sensibilidade & 62,5 & $5,9 \quad 8$ & 83,7 & Sensibilidade & 62,5 & 35,9 & 83,7 & Sensibilidade & 66,7 & 35,4 & 88,7 & Sensibilidade & 68,2 & 45,1 & 85,3 & Sensibilidade & 64,0 & 42,6 & 81,3 \\
\hline Especificidade & 87,5 & 70,1 & 95,9 & Especificidade & 87,5 & 70,1 & 95,9 & Especificidade & 97,2 & 83,8 & 99,9 & Especificidade & 80,8 & 60,0 & 92,7 & Especificidade & 78,3 & 55,8 & 91,7 \\
\hline VPP & 71,4 & 42,0 & 90,4 & VPP & 71,4 & 42,0 & 90,4 & VPP & 88,9 & 50,7 & 99,4 & VPP & 75,0 & 50,6 & 90,4 & VPP & 76,2 & 52,4 & 90,9 \\
\hline VPN & 82,4 & 64,2 & 92,6 & VPN & 82,4 & 64,2 & 92,6 & VPN & 89,7 & 74,8 & 96,7 & VPN & 75,0 & 54,8 & 88,6 & VPN & 66,7 & 46,0 & 82,8 \\
\hline Prev. F & 33,3 & 24,1 & 42,5 & Prev. F & 33,3 & 24,1 & 42,2 & Prev. F & $25,0 \quad 1$ & 16,5 & 33,5 & Prev. F & 45,8 & 36,0 & 55,6 & Prev. F & 52,1 & 42,3 & 61,9 \\
\hline Prev. R & 29,2 & 20,3 & 38,1 & Prev. R & 29,2 & 20,3 & 38,1 & Prev. R & 18,8 & 11,1 & 26,5 & Prev. R & 41,7 & 32,0 & 51,4 & Prev. R & 43,8 & 34,1 & 53,5 \\
\hline Prev. F e/ou R & 41,7 & $32,0=$ & 51,4 & Prev. F e/ou R & 41,7 & 32,0 & 51,4 & Prev. F e/ou R & 27,1 & 18,4 & 35,8 & Prev. F e/ou R & 56,3 & 46,6 & 66,0 & Prev. F e/ou R & 62,5 & 53,0 & 72,0 \\
\hline Acurácia & 79,2 & 71,2 & 87,2 & Acurácia & 79,2 & 71,2 & 87,2 & Acurácia & $89,6 \quad 8$ & 83,6 & 95,6 & Acurácia & 75,0 & 66,5 & 83,5 & Acurácia & 70,8 & 61,9 & 79,7 \\
\hline $\begin{array}{c}\text { Qui } \\
\text { quadrado }\end{array}$ & $\chi^{2}$ & valor d & de $\mathrm{p}$ & $\begin{array}{c}\text { Qui } \\
\text { quadrado }\end{array}$ & $\chi^{2}$ & val & $p$ & $\begin{array}{c}\text { Qui } \\
\text { quadrado }\end{array}$ & $\chi^{2}$ & valor & $\mathrm{rde} p$ & $\begin{array}{c}\text { Qui } \\
\text { quadrado }\end{array}$ & $\chi^{2}$ & valor & le p & Qui Quadrado & $\chi^{2}$ & $c^{2}$ & valor de $\mathrm{p}$ \\
\hline Não corrigido & $12,91 \quad 0$ & 0,00032 & 32726 & Não corrigido & 12,91 & 0,000 & 2726 & Não corrigido & 24,11 & 0,000 & 00091 & Não corrigido & 11,75 & 0,000 & 60900 & Não corrigido & 8,6 & 69 & 0,00319318 \\
\hline M-H & $12,64 \quad 0$ & 0,00037 & 37785 & M-Hl & 12,64 & 0,000 & 37785 & $\mathrm{M}-\mathrm{H}$ & 23,61 & 0,000 & 00118 & $\mathrm{M}-\mathrm{H}$ & 11,50 & 0,000 & 69465 & M-H & 8,5 & 51 & 0,00352706 \\
\hline $\begin{array}{l}\text { Yates } \\
\text { corrigido }\end{array}$ & $10,60 \quad 0$ & 0,00113 & 3036 & $\begin{array}{l}\text { Yates } \\
\text { corrigido }\end{array}$ & 10,60 & 0,001 & 3036 & $\begin{array}{l}\text { Yates } \\
\text { corrigido }\end{array}$ & 20,10 & 0,000 & 00734 & $\begin{array}{l}\text { Yates } \\
\text { corrigido }\end{array}$ & 9,82 & 0,001 & 72570 & $\begin{array}{l}\text { Yates } \\
\text { corrigido }\end{array}$ & 7,0 & 06 & 0,00787712 \\
\hline \multirow[t]{2}{*}{$\begin{array}{l}\text { Fisher exato } \\
\mathrm{p}=\end{array}$} & \multirow{2}{*}{\multicolumn{3}{|c|}{ Unimodal }} & $\begin{array}{l}\text { Fisher exato } \\
\mathrm{p}=\end{array}$ & \multicolumn{3}{|c|}{ unimodal bimodal } & $\begin{array}{l}\text { Fisher exato } \\
\mathrm{p}=\end{array}$ & \multicolumn{3}{|c|}{ Unimodal bimodal } & & & & & & & & \\
\hline & & & & & 0,000643 & 1390 , & 006439 & & 0,0000108 & $8 \quad 0,0$ & 000108 & & & & & & & & \\
\hline Coeficiente $\mathrm{K}$ & \multicolumn{3}{|c|}{0,516129} & Coeficiente Қ & \multicolumn{3}{|c|}{0,516129} & Coeficiente $\mathrm{K}$ & \multicolumn{3}{|c|}{0,696970} & Coeficiente $\mathrm{K}$ & \multicolumn{3}{|c|}{0,492958} & Coeficiente K & \multicolumn{3}{|c|}{0,419689} \\
\hline $\begin{array}{l}\text { Catadores } \\
\text { Pos. }\end{array}$ & \multicolumn{3}{|c|}{20} & $\begin{array}{l}\text { Catadores } \\
\text { Pos. }\end{array}$ & \multicolumn{3}{|c|}{20} & $\begin{array}{l}\text { Catadores } \\
\text { Pos. }\end{array}$ & \multicolumn{3}{|c|}{13} & $\begin{array}{l}\text { Catadores } \\
\text { Pos. }\end{array}$ & \multicolumn{3}{|c|}{27} & $\begin{array}{l}\text { Catadores } \\
\text { Pos. }\end{array}$ & & & 30 \\
\hline
\end{tabular}

Ritchie $_{1}$ : Ritchie $1^{\text {a }}$ amostra; Ritchie ${ }_{2}$ : Ritchie $2^{\text {a }}$ amostra; Ritchie ${ }_{3}$ : Ritchie $3^{\text {a }}$ amostra; Ritchie ${ }_{11}$ : Ritchie $1^{\text {a }}$ e $2^{\text {a }}$ amostras; Ritchie ${ }_{123}$ : Ritchie $1^{\text {a }}, 2^{\mathrm{a}}$ e $3^{\mathrm{a}}$ amostras; Faust ${ }_{1}$ : Faust $1^{\mathrm{a}}$ amostra; Faust ${ }_{2}$ : Faust $2^{\mathrm{a}}$ amostra; Faust ${ }_{3}$ : Faust $3^{\mathrm{a}}$ amostra; Faust ${ }_{12}$ : Faust $1^{\mathrm{a}}$ e $2^{\mathrm{a}}$ amostras; Faust $_{123}$ : Faust 1 $1^{\text {a }} 2^{\text {a }}$ e $3^{\text {a }}$ amostras; M-H: Mantel-Haenszel; I.C. 95\%: Intervalo de Confiança; Pos: Positivo; Neg: Negativo; Li: Limite inferior; Ls: Limite superior; VPP: Valor Preditivo Positivo; VPN: Valor Preditivo Negativo; Prev.: Prevalência.

poliparasitados, as medidas de efeito podem ser verificadas nas Tabelas 2.7 à 2.9 .

\section{Helmintos}

Trichuris trichiura foi o helminto mais detectado com $22,92 \%$ de frequência, sendo $72,72 \%(8 / 11)$ por Faust e 54,55\% (6/11) por Ritchie. A coincidência em ambas as técnicas detectou $27,27 \%$ (3/11). As medidas de efeito de Ritchie em relação à Faust podem ser visualizadas na Tabela 2.10.

Em relação à detecção de $T$. trichiura, na $1^{\mathrm{a}}$ amostra foram detectados $27,3 \%(3 / 11)$, na $2^{\mathrm{a}}$ detectaram-se $54,5 \%(6 / 11)$ e na $3^{\text {a }}$ detectaram-se $45,5 \%(5 / 11)$. Se fosse considerada somente uma técnica parasitológica, na $2^{\mathrm{a}}$ ou na $3^{\mathrm{a}}$ coleta é que se conseguiria o maior número de parasitados, com $36,4 \%$ do total encontrado. Entre os parasitados o percentual de detecção foi de $36,67 \%$ (11/30) (Tabelas 3.1 à 3.4).

O segundo helminto mais prevalente foi Ascaris lumbricoides $\quad(18,75 \%)$, sendo $88,89 \%$ diagnosticado com Ritchie e 77,78\% (7/9) com Faust. Ambas as técnicas coincidiram na detecção em 66,67\% (6/9). As medidas de efeito de Faust em relação a Ritchie podem ser visualizadas na Tabela 2.11.

Em relação à detecção de $A$. lumbricoides nas coletas individuais, os percentuais alcançados foram de $77,78 \%(7 / 9)$ na $1^{\text {a }}$ coleta, $55,56 \%(5 / 9)$ na $2^{\text {a }}$ coleta e de $55,6 \%(5 / 9)$ na $3^{\text {a }}$ coleta. Entre os parasitados, o percentual de detecção foi de 30,0\% (9/30) (Tabelas 3.5 à 3.8$)$.

Em terceiro lugar, o helminto Strongyloides stercoralis, teve uma prevalência de $10,42 \%$, sendo $80 \%$ (4/5) diagnosticado por Ritchie e 20\% (1/5) por Faust. Não houve correlação positiva entre as técnicas. As medidas de efeito de Faust em relação a Ritchie podem ser visualizadas na Tabela 2.12.

S. stercoralis, na sua sequência de coletas os percentuais de deteç̧ão alcançados foram de 20,0\% $(1 / 5)$ na $1^{\text {a }}$ coleta, $80,0 \%(4 / 5)$ na $2^{\text {a }}$ coleta e de $20,0 \%$ $(1 / 5)$ na $3^{\text {a }}$ coleta. Entre os parasitados o percentual de detecção foi de $16,67 \%(5 / 30)$ (Tabelas 3.9 à 3.12). 
Tabela 2 - Análise das medidas de efeito das técnicas de Faust e Ritchie para os enteroparasitos encontrados em 48 catadores de materiais recicláveis das seis cooperativas de triagem de resíduos sólidos do Município de Pelotas (RS) entre maio e dezembro de 2016.

\begin{tabular}{|c|c|c|c|c|c|c|c|c|c|c|c|c|c|c|c|c|c|c|c|c|c|c|c|}
\hline \multirow{3}{*}{$\begin{array}{c}2.1 \\
\text { Ritchie }\end{array}$} & \multirow{2}{*}{\multicolumn{3}{|c|}{$\begin{array}{l}\text { Parasitados } \\
\text { Faust }\end{array}$}} & \multirow{2}{*}{$\begin{array}{c}\text { IC95\% } \\
\text { S }\end{array}$} & \multirow{3}{*}{$\begin{array}{l}64,0 \\
78,3\end{array}$} & \multirow{3}{*}{$\begin{array}{c}\mathbf{L}_{\mathbf{i}} \\
42,6 \\
55,8\end{array}$} & \multirow{3}{*}{$\begin{array}{c}\mathbf{L}_{\mathrm{s}} \\
81,3 \\
91,7\end{array}$} & \multirow{3}{*}{$\begin{array}{c}2.2 \\
\text { Ritchie }\end{array}$} & \multirow{2}{*}{\multicolumn{3}{|c|}{$\begin{array}{l}\text { Protozoários } \\
\text { Faust }\end{array}$}} & \multirow{2}{*}{$\begin{array}{c}\text { IC95\% } \\
\mathrm{S}\end{array}$} & \multirow{3}{*}{$\begin{array}{l}35,7 \\
97,1\end{array}$} & \multirow{3}{*}{$\begin{array}{c}\mathbf{L}_{\mathbf{i}} \\
14,0 \\
82,9\end{array}$} & \multirow{3}{*}{$\begin{array}{c}\mathbf{L}_{\mathrm{s}} \\
64,4 \\
99,8\end{array}$} & \multirow{3}{*}{$\begin{array}{c}2.3 \\
\text { Ritchie }\end{array}$} & \multirow{2}{*}{\multicolumn{3}{|c|}{$\begin{array}{l}\text { Helmintos } \\
\text { Faust }\end{array}$}} & \multirow{2}{*}{$\begin{array}{c}\text { IC95\% } \\
\mathrm{S}\end{array}$} & \multirow{3}{*}{$\begin{array}{l}55,6 \\
80,0\end{array}$} & \multirow{3}{*}{$\begin{array}{c}\mathbf{L}_{\mathbf{i}} \\
31,3 \\
60,9\end{array}$} & \multirow{3}{*}{$\begin{array}{c}\mathbf{L}_{\mathrm{s}} \\
77,6 \\
91,6\end{array}$} \\
\hline & & & & & & & & & & & & & & & & & & & & & & & \\
\hline & Pos. & Neg. & Tot. & $\mathbf{E}$ & & & & & Pos. & Neg. & Tot. & E & & & & & Pos. & Neg. & Tot. & $\mathbf{E}$ & & & \\
\hline Pos. & 16 & 5 & 21 & VPP & 76,2 & 52,4 & 90,9 & Pos. & 5 & 1 & 6 & VPP & 83,3 & 82,9 & 99,1 & Pos. & 10 & 6 & 16 & VPP & 62,5 & 35,9 & 83,7 \\
\hline Neg. & 9 & 18 & 27 & VPN & 66,7 & 46,0 & 82,8 & Neg. & 9 & 33 & 33 & VPN & 78,6 & 62,8 & 89,2 & Neg. & 8 & 24 & 32 & VPN & 75,0 & 56,2 & 87,9 \\
\hline Tot. & 25 & 23 & 48 & К & 0,76 & & & Tot. & 14 & 34 & 48 & Қ & 0,393 & & & Tot. & 18 & 30 & 48 & қ & 0,363 & & \\
\hline \multirow[t]{2}{*}{2.4} & Mono & parasi & $\operatorname{tados}$ & IC95\% & & $\mathbf{L}_{\mathbf{i}}$ & $\mathbf{L}_{\mathrm{s}}$ & 2.5 & Prot & zoário & s MP & IC95\% & & $\mathbf{L}_{\mathrm{i}}$ & $\mathbf{L}_{\mathrm{s}}$ & 2.6 & Hel & mintos & MP & IC95\% & & $\mathbf{L}_{\mathrm{i}}$ & $\mathbf{L}_{\mathrm{s}}$ \\
\hline & $\mathbf{F a}$ & ust & & $\mathbf{S}$ & 33,3 & 11,3 & 64,6 & & & ust & & S & 25,0 & 1,3 & 78,1 & & & lust & & $\mathbf{S}$ & 37,5 & 10,2 & 74,1 \\
\hline Ritchie & Pos. & Neg. & Tot. & $\mathbf{E}$ & 86,1 & 69,7 & 94,8 & Ritchie & Pos. & Neg. & Tot. & E & 97,7 & 86,5 & 99,9 & Ritchie & Pos. & Neg. & Tot. & $\mathbf{E}$ & 90,0 & 75,4 & 96,7 \\
\hline Pos. & 4 & 5 & 9 & VPP & 44,4 & 15,3 & 77,3 & Pos. & 1 & 1 & 2 & VPP & 50,0 & 2,7 & 97,3 & Pos. & 3 & 4 & 7 & VPP & 42,9 & 11,8 & 79,8 \\
\hline Neg. & 8 & 31 & 39 & VPN & 79,5 & 63,1 & 90,1 & Neg. & 3 & 43 & 46 & VPN & 93,5 & 81,1 & 98,3 & Neg. & 5 & 36 & 41 & VPN & 87,8 & 73,0 & 95,4 \\
\hline Tot. & 12 & 36 & 48 & қ & 0,21 & & & Tot. & 4 & 44 & 48 & К & 0,29 & & & Tot. & 8 & 40 & 48 & қ & 0,29 & & \\
\hline 2.7 & Polip & ast & ados & IC95\% & & $\mathbf{L}_{\mathrm{i}}$ & $\mathbf{L}_{\mathrm{s}}$ & 2.8 & Proto & zoário & PP & IC95\% & & $\mathbf{L}_{\mathrm{i}}$ & $\mathbf{L}_{\mathrm{s}}$ & 2.9 & Hel & ов & $P$ & IC95\% & & $\mathbf{L}_{\mathrm{i}}$ & $\mathbf{L}_{\mathrm{s}}$ \\
\hline Ritchie & Pos. & Neg. & Tot. & $\mathbf{E}$ & 100,0 & 87,7 & 100,0 & Ritchie & Pos. & Neg. & Tot. & E & 100,0 & 88,6 & 100,0 & Ritchie & Pos. & Neg. & Tot. & $\mathbf{E}$ & 94,7 & 80,9 & 99,1 \\
\hline Pos. & 12 & 0 & 12 & VPP & 100,0 & 69,9 & 100,0 & Pos. & 4 & 0 & 4 & VPP & 100,0 & 39,6 & 100,0 & Pos. & 7 & 2 & 9 & VPP & 77,8 & 40,2 & 96,1 \\
\hline Neg. & 1 & 35 & 36 & VPN & 97,2 & 83,8 & 99,9 & Neg. & 6 & 38 & 44 & VPN & 86,4 & 72,0 & 94,3 & Neg. & 3 & 36 & 39 & VPN & 92,3 & 78,0 & 98,0 \\
\hline Tot. & 13 & 35 & 48 & қ & 0,95 & & & Tot. & 10 & 38 & 48 & К & 0,51 & & & Tot. & 10 & 38 & 48 & қ & 0,67 & & \\
\hline 2.10 & & $T t$ & & IC95\% & & $\mathbf{L}_{\mathrm{i}}$ & $\mathbf{L}_{\mathrm{s}}$ & 2.11 & & $A l$ & & IC95\% & & $\mathbf{L}_{\mathrm{i}}$ & $\mathbf{L}_{\mathrm{s}}$ & 2.12 & & Ss & & IC95\% & & $\mathbf{L}_{\mathrm{i}}$ & $\mathbf{L}_{\mathrm{s}}$ \\
\hline & $\mathrm{Fa}$ & ust & & $\mathbf{S}$ & 37,5 & 10,2 & 74,5 & & & chie & & $\mathbf{S}$ & 75,55 & 35,6 & 95,5 & & & chie & & S & 0,0 & 0,0 & 60,4 \\
\hline Ritchie & Pos. & Neg. & Tot. & $\mathbf{E}$ & 92,5 & 78,5 & 98,0 & Faust & Pos. & Neg. & Tot. & E & 97,5 & 85,3 & 99,9 & Faust & Pos. & Neg. & Tot. & $\mathbf{E}$ & 97,7 & 86,5 & 99,9 \\
\hline Pos. & 3 & 3 & 6 & VPP & 50,0 & 13,9 & 86 & Pos. & 6 & 1 & 7 & VPP & 85,7 & 42,0 & 99,2 & Pos. & 0 & 1 & 1 & VPP & 0,0 & 0,0 & 94,5 \\
\hline Neg. & 5 & 37 & 42 & VPN & 88,1 & 73,6 & 95,5 & Neg. & 2 & 39 & 41 & VPN & 95,1 & 82,2 & 99,2 & Neg. & 4 & 43 & 44 & VPN & 91,5 & 78,7 & 97,2 \\
\hline Tot. & 8 & 40 & 48 & қ & 0,33 & & & Tot. & 8 & 40 & 48 & К & 0,76 & & & Tot. & 4 & 44 & 48 & қ & $-0,03$ & & \\
\hline 2.13 & & $T s p$ & & IC95\% & & $\mathbf{L}_{\mathrm{i}}$ & $\mathbf{L}_{\mathrm{s}}$ & 2.14 & & $H n$ & & IC95\% & & $\mathbf{L}_{\mathbf{i}}$ & $\mathbf{L}_{\mathrm{s}}$ & 2.15 & & $E c$ & & IC95\% & & $\mathbf{L}_{\mathrm{i}}$ & $\mathbf{L}_{\mathrm{s}}$ \\
\hline & & ust & & $\mathbf{S}$ & 25,0 & 1,3 & 78., & & & ust & & $\mathbf{S}$ & 0,0 & 0,0 & 94,5 & & & ust & & S & 30,0 & 8,1 & 64,6 \\
\hline Ritchie & Pos. & Neg. & Tot. & T & 100,0 & 90,0 & 100,0 & chie & Pos. & Neg. & Tot. & $\Gamma$ & 97,9 & 87,3 & 99,9 & hie & Pos. & Neg. & Tot. & $\Gamma$ & 94,7 & 80,9 & 99,1 \\
\hline Tot. & 4 & 44 & 48 & қ & 0,38 & & & Tot. & 1 & 47 & 48 & Қ & $-0,02$ & & & Tot. & 10 & 38 & 48 & қ & 0,30 & & \\
\hline 2.16 & & En & & IC95\% & & $\mathbf{L}_{\mathrm{i}}$ & $\mathbf{L}_{\mathrm{s}}$ & 17 & & $E \boldsymbol{h}$ & & IC95\% & & $L_{i}$ & $\mathbf{L}_{\mathrm{s}}$ & 10 & & $G l$ & & IC95\% & & $L_{i}$ & $\mathbf{L}_{\mathrm{s}}$ \\
\hline & & ust & & $\mathbf{S}$ & 0,0 & & & & & chie & & $\mathbf{S}$ & 0,0 & & & & & lust & & $\mathbf{S}$ & 50,0 & 2,7 & 97,3 \\
\hline Ritchie & Pos. & Neg. & Tot. & $\mathbf{E}$ & 0,0 & & & Faust & Pos. & Neg. & Tot. & E & 0,0 & & & Ritchie & Pos. & Neg. & Tot. & $\mathbf{E}$ & 100,0 & 90,4 & 100,0 \\
\hline Pos. & 0 & 0 & 0 & VPP & 0,0 & & & Pos. & 0 & 0 & 0 & VPP & 0,0 & & & Pos. & 1 & 0 & 1 & VPP & 100,0 & 5,5 & 100,0 \\
\hline Neg. & 5 & 43 & 48 & VPN & 0,0 & & & Neg. & 2 & 46 & 48 & VPN & 0,0 & & & Neg. & 1 & 46 & 47 & VPN & 97,9 & 87,3 & 99,9 \\
\hline Tot. & 5 & 43 & 48 & n & 0,0 & & & Tot. & 2 & 46 & 48 & 4 & 0,0 & & & Tot. & 2 & 46 & 48 & қ & 0,66 & & \\
\hline
\end{tabular}

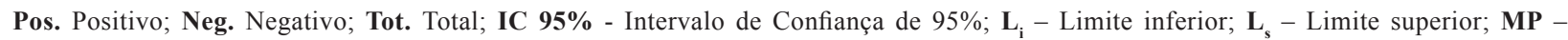
Monoparasitose; PP - Poliparasitose; S: Sensibilidade; E: Especificidade; VPP: Valor Preditivo Positivo; VPN: Valor Preditivo Negativo; Қ: Coeficiente de Correlação Kappa; Al: Ascaris lumbricoides; Tt: Trichuris trichiura; Ss: Strongyloides stercoralis; Tsp: Taenia sp; Hn: Hymenolepis nana; Ec: Entamoeba coli; En: Endolimax nana;Eh: Entamoeba hartmanni;Gl: Giardia lamblia.

Em quarto lugar o helminto Taenia sp. foi detectado com uma prevalência de $8,33 \%$, sendo $100,0 \%$ (4/4) por Faust e 25,0\% (1/4) também por Ritchie. As medidas de efeito de Ritchie em relação à Faust podem ser visualizadas na Tabela 2.13. Nas coletas sequenciais os percentuais de detecção alcançados foram de $75,0 \%$ (3/4) na $1^{\mathrm{a}}$ coleta, $75,0 \%(3 / 4)$ na $2^{\mathrm{a}}$ coleta e de $25,0 \%$ (1/4) na $3^{\text {a }}$ coleta. Entre os parasitados o percentual de detecção foi de 13,33\% (4/30) (Tabelas 3.13 à 3.16).

Por fim, em quinto lugar, o helminto Hymenolepis nana foi diagnosticado com uma prevalência de $4,17 \%$, sendo detectado em $50,0 \%(1 / 2)$ por Faust e $50,0 \%$ $(1 / 2)$ por Ritchie. Não houve correlação positiva entre as técnicas, Tabela 2.14. Nas coletas sequenciais os percentuais de detecção alcançados foram de 50,0\% $(1 / 2)$ por Ritchie na $1^{\text {a }}$ coleta, $50,0 \%(1 / 2)$ por Faust na $2^{\text {a }}$ coleta e de $0,0 \%(0 / 2)$ na $3^{\text {a }}$ coleta. Entre os parasitados o percentual de detecção foi de $6,67 \%$ $(2 / 30)$ (Tabelas 3.17 à 3.20).

O total de helmintos detectados nas amostras sequenciais foi de $54,17 \%(13 / 24)$ na $1^{\text {a }}$ coleta, de $70,83 \%(17 / 24)$ na $2^{\text {a }}$ coleta e de $45,83 \%(11 / 24)$ na 
$3^{\text {a }}$ coleta, totalizando $80,0 \%(24 / 30)$ dos parasitados (Tabelas 3.21 à 3.24).

\section{Protozoários}

O protozoário detectado em um maior número de coletores de materiais recicláveis foi Entamoeba coli (25,00\%), sendo detectado em 83,33\% 10/12) por Faust e em 41,66\% (5/12) por Ritchie. Ambas as técnicas coincidiram na detecção em 25,00\%(3/12). As medidas de efeito de Ritchie em relação à Faust constam na Tabela 2.15. Nas coletas sequenciais os percentuais de detecção alcançados foram de $66,67 \%$ $(8 / 12)$ na $1^{\text {a }}$ coleta, $41,67 \%(5 / 12)$ na $2^{\text {a }}$ coleta e de $50,0 \%(6 / 12)$ na $3^{\text {a }}$ coleta. Entre os parasitados o percentual de detecção foi de 40,0\%(12/30) (Tabelas 3.25 à 3.28).

Em segundo lugar, a prevalência do protozoário Endolimax nana foi de 10,45\%, sendo 100,0\% (5/5) identificado por Faust e 0,0\% (0/5) por Ritchie (Tabela 2.16). Nas coletas sequenciais os percentuais de detecção alcançados foram de $40,0 \%(2 / 5)$ na $1^{\text {a }}$ coleta, $40,0 \%(2 / 5)$ na $2^{\mathrm{a}}$ coleta e de $20,0 \%(1 / 5)$ na $3^{\mathrm{a}}$

Tabela 3 - Diagnóstico de Helmintos e Protozoários por Faust e Ritchie em 3 amostras de fezes de 48 catadores de material reciclável das seis Cooperativas do Município de Pelotas (RS) entre maio e dezembro de 2016.

\begin{tabular}{|c|c|c|c|c|c|c|c|c|c|c|c|c|c|c|c|c|c|c|c|c|c|c|c|c|c|c|c|c|c|c|c|}
\hline \multicolumn{16}{|c|}{ HELMINTOS } & \multicolumn{16}{|c|}{ PROTOZOÁRIOS } \\
\hline \multicolumn{4}{|c|}{ Amostra 1} & \multicolumn{4}{|c|}{ Amostra 2} & \multicolumn{4}{|c|}{ Amostra 3} & \multicolumn{4}{|c|}{3 Amostras } & \multicolumn{4}{|c|}{ Amostra 1} & \multicolumn{4}{|c|}{ Amostra 2} & \multicolumn{4}{|c|}{ Amostra 3} & \multicolumn{4}{|c|}{3 Amostras } \\
\hline 3.1 & \multicolumn{2}{|c|}{$\mathrm{R}_{1} T t$} & \multirow[b]{2}{*}{$\mathbf{T}$} & \multirow{2}{*}{3.2} & \multicolumn{2}{|c|}{$\mathrm{R}_{2} T t$} & \multirow[b]{2}{*}{$\mathbf{T}$} & 3.3 & & $T t$ & & 3.4 & Par & cas. & & 3.25 & $\mathbf{R}_{1}$ & $E c$ & & 3.26 & $\mathbf{R}_{2}$ & & & 3.27 & $\mathbf{R}_{3}$ & & & 3.28 & Paras & & \\
\hline $\mathrm{F}_{1} T t$ & $\mathbf{P}$ & $\mathbf{N}$ & & & $\mathbf{P}$ & $\mathbf{N}$ & & $\mathrm{F}_{3} T t$ & $\mathrm{P}$ & $\mathrm{N}$ & $\mathrm{T}$ & $\mathrm{FR} T t$ & $\mathrm{P}$ & $\mathrm{N}$ & $\mathrm{T}$ & $F_{1} E c$ & $\mathrm{P}$ & $\mathrm{N}$ & $\mathrm{T}$ & $\mathrm{F}_{2} E c$ & $\mathrm{P}$ & $\mathrm{N}$ & $\mathrm{T}$ & $\mathrm{F}_{3} E c$ & $\mathrm{P}$ & $\mathrm{N}$ & $\mathrm{T}$ & FREc & $\mathrm{P} \quad \mathrm{N}$ & & $\mathrm{T}$ \\
\hline $\mathbf{P}$ & 1 & 1 & 2 & $\mathbf{P}$ & 2 & 2 & 4 & $\mathrm{P}$ & 2 & 2 & 4 & $P$ & 11 & & 11 & $P$ & 3 & 3 & 6 & $P$ & 1 & 3 & 4 & $P$ & 1 & & 6 & $P$ & 120 & 0 & 12 \\
\hline $\mathbf{N}$ & 1 & 45 & 46 & $\mathbf{N}$ & 2 & 42 & 44 & $\mathrm{~N}$ & 1 & 43 & 44 & $\mathrm{~N}$ & 19 & 18 & 37 & $\mathrm{~N}$ & 2 & 40 & 42 & $\mathrm{~N}$ & 1 & 43 & 44 & $\mathrm{~N}$ & 0 & 42 & 42 & $\mathrm{~N}$ & $18 \quad 1$ & 18 & 36 \\
\hline $\mathbf{T}$ & 2 & 46 & 48 & $\mathbf{T}$ & 4 & 44 & 48 & $\mathrm{~T}$ & 3 & 45 & 48 & $\mathrm{~T}$ & 30 & 18 & 48 & $\mathrm{~T}$ & 5 & 43 & 48 & $\mathrm{~T}$ & 2 & 46 & 48 & $\mathrm{~T}$ & 1 & 47 & 48 & $\mathrm{~T}$ & $\begin{array}{ll}30 & 1\end{array}$ & 18 & 48 \\
\hline $\mathrm{F}_{1} A l$ & $\mathrm{P}$ & $\mathrm{N}$ & $\mathrm{T}$ & $\mathrm{F}_{2} A l$. & $\mathrm{P}$ & $\mathrm{N}$ & $\mathrm{T}$ & $\mathrm{F}_{3} A l$ & $\mathrm{P}$ & $\mathrm{N}$ & $\mathrm{T}$ & FR $A l$ & $\mathrm{P}$ & $\mathrm{N}$ & $\mathrm{T}$ & $F_{1} E n$ & $\mathrm{P}$ & $\mathrm{N}$ & $\mathrm{T}$ & $\mathrm{F}_{2} E n$ & $\mathrm{P}$ & $\mathrm{N}$ & $\mathrm{T}$ & $\mathrm{F}_{3} E n$ & $\mathrm{P}$ & $\mathrm{N}$ & $\mathrm{T}$ & FREn & $\begin{array}{ll}\mathrm{P} & \mathrm{N} \\
\end{array}$ & $\mathrm{N}$ & $\mathrm{T}$ \\
\hline $\mathrm{P}$ & 4 & 1 & 5 & $P$ & 3 & 0 & 3 & $\mathrm{P}$ & 3 & 1 & 4 & $\mathrm{P}$ & 9 & & 9 & $\mathrm{P}$ & 0 & & 2 & $\mathrm{P}$ & & & 2 & $\mathrm{P}$ & & & 1 & $\mathrm{P}$ & 50 & & 5 \\
\hline $\mathrm{N}$ & 2 & 41 & 43 & $\mathrm{~N}$ & 2 & 43 & 45 & $\mathrm{~N}$ & 1 & 43 & 44 & $\mathrm{~N}$ & 21 & 18 & 39 & $\mathrm{~N}$ & 0 & 46 & 46 & $\mathrm{~N}$ & & 46 & 46 & $\mathrm{~N}$ & 0 & 47 & 47 & $\mathrm{~N}$ & $25 \quad 1$ & 18 & 43 \\
\hline $\mathrm{T}$ & 6 & 42 & 48 & $\mathrm{~T}$ & 5 & 43 & 48 & $\mathrm{~T}$ & 4 & 44 & 48 & $\mathrm{~T}$ & 30 & 18 & 48 & $\mathrm{~T}$ & 0 & 48 & 48 & $\mathrm{~T}$ & 0 & 48 & 48 & $\mathrm{~T}$ & 0 & 48 & 48 & $\mathrm{~T}$ & $30 \quad 1$ & $\overline{18}$ & 48 \\
\hline 3.9 & $\mathbf{R}_{1^{\prime}}$ & Ss & & 3.10 & $\mathbf{R}_{2}$ & & & 3.11 & & Ss & & 3.12 & Par & cas. & & 3.33 & $\mathbf{R}_{1}$ & $E h$ & & 3.34 & $\mathbf{R}_{2} 1$ & $E h$ & & 3.35 & $\mathbf{R}_{3} \mathbf{I}$ & $E h$ & & 3.36 & Paras & as. & \\
\hline$F_{1} S s$ & $\mathrm{P}$ & $\mathrm{N}$ & $\mathrm{T}$ & $\mathrm{F}_{2} S s$ & $\mathrm{P}$ & $\mathrm{N}$ & $\mathrm{T}$ & $\mathrm{F}_{3} S \boldsymbol{s}$ & $\mathrm{P}$ & $\mathrm{N}$ & $\mathrm{T}$ & FRSs & $\mathrm{P}$ & $\mathrm{N}$ & $\mathrm{T}$ & $F_{1} E h$ & $\mathrm{P}$ & $\mathrm{N}$ & $\mathrm{T}$ & $\mathbf{F}_{2} E h$ & $\mathrm{P}$ & $\mathrm{N}$ & $\mathrm{T}$ & $\mathbf{F}_{3} E \boldsymbol{h}$ & $\mathrm{P}$ & $\mathrm{N}$ & $\mathrm{T}$ & FREh & $\begin{array}{ll}P & N\end{array}$ & $\mathrm{~N}$ & $\mathrm{~T}$ \\
\hline $\mathrm{N}$ & 1 & 47 & 48 & $\mathrm{~N}$ & 3 & 44 & 47 & $\mathrm{~N}$ & 1 & 47 & 48 & $\mathrm{~N}$ & 25 & 18 & 43 & $\mathrm{~N}$ & 2 & 46 & 48 & $\mathrm{~N}$ & 0 & 48 & 48 & $\mathrm{~N}$ & 0 & 48 & 48 & $\mathrm{~N}$ & $28 \quad 1$ & 18 & 46 \\
\hline $\mathrm{T}$ & 1 & 47 & 48 & $\mathrm{~T}$ & 3 & 45 & 48 & $\mathrm{~T}$ & 1 & 47 & 48 & $\mathrm{~T}$ & 30 & 18 & 48 & $\mathrm{~T}$ & 2 & 46 & 48 & $\mathrm{~T}$ & 0 & 48 & 48 & $\mathrm{~T}$ & 0 & 48 & 48 & $\mathrm{~T}$ & $30 \quad 1$ & $\overline{18}$ & 48 \\
\hline 3.13 & $\mathbf{R}_{1}$ & $T s p$ & & 3.14 & $\mathbf{R}_{2}$ & & & 3.15 & & $T s p$ & & 3.16 & Par & cas. & & 3.37 & $\mathbf{R}_{1}$ & $G l$ & & 3.38 & $\mathbf{R}_{2}$ & & & 3.39 & $\mathbf{R}_{3}$ & $G l$ & & 3.40 & Paras & & \\
\hline $\mathrm{F}_{1} T_{s p}$ & $\mathrm{P}$ & $\mathrm{N}$ & $\mathrm{T}$ & $\mathrm{F}_{2} T_{s p} p$ & $\mathrm{P}$ & $\mathrm{N}$ & $\mathrm{T}$ & $\mathrm{F}_{3} T s p$ & $\mathrm{P}$ & $\mathrm{N}$ & $\mathrm{T}$ & FRT $T s p$ & $\mathrm{P}$ & $\mathrm{N}$ & $\mathrm{T}$ & $\mathrm{F}_{1} G l$ & $\mathrm{P}$ & $\mathrm{N}$ & $\mathrm{T}$ & $\mathrm{F}_{2} G l$ & $\mathrm{P}$ & $\mathrm{N}$ & $\mathrm{T}$ & $\mathrm{F}_{3} G l$ & $\mathrm{P}$ & $\mathrm{N}$ & $\mathrm{T}$ & FRGl & $\begin{array}{ll}\mathrm{P} & \mathrm{N} \\
\end{array}$ & $\mathrm{N}$ & $\mathrm{T}$ \\
\hline $\mathrm{P}$ & 0 & 3 & 3 & $\mathrm{P}$ & 0 & 3 & 3 & $P$ & 1 & 0 & 1 & $\mathrm{P}$ & 4 & & 4 & $\mathrm{P}$ & 0 & & 0 & $\mathrm{P}$ & 1 & & 1 & $\mathrm{P}$ & 0 & & 1 & $\mathrm{P}$ & 20 & & 2 \\
\hline $\mathrm{N}$ & 0 & 45 & 45 & $\mathrm{~N}$ & 0 & 45 & 45 & $\mathrm{~N}$ & 0 & 47 & 47 & $\mathrm{~N}$ & 26 & 18 & 44 & $\mathrm{~N}$ & 0 & 48 & 48 & $\mathrm{~N}$ & 0 & 47 & 47 & $\mathrm{~N}$ & 0 & 47 & 47 & $\mathrm{~N}$ & $28 \quad 1$ & 18 & 46 \\
\hline $\mathrm{T}$ & 0 & 48 & 48 & $\mathrm{~T}$ & 0 & 48 & 48 & $\mathrm{~T}$ & 1 & 47 & 48 & $\mathrm{~T}$ & 30 & 18 & 48 & $\mathrm{~T}$ & 0 & 48 & 48 & $\mathrm{~T}$ & 1 & 47 & 48 & $\mathrm{~T}$ & 0 & 48 & 48 & $\mathrm{~T}$ & $30 \quad 1$ & 18 & 48 \\
\hline 3.17 & $\mathbf{R}_{1}$ & & & 3.18 & $\mathbf{R}_{2}$ & & & 3.19 & & $H n$ & & 3.20 & Par & cas. & & 3.41 & $F_{1}$ & TP & & 3.42 & $\mathbf{F}_{2} \mathbf{T}$ & & & 3.43 & $\mathbf{F}_{3} \mathbf{T}$ & ТP & & 3.44 & FTP & & \\
\hline $\mathrm{F}_{1} \mathrm{Hn}$ & $\mathrm{P}$ & $\mathrm{N}$ & $\mathrm{T}$ & $\mathrm{F}_{2} \mathrm{Hn}$ & $\mathrm{P}$ & $\mathrm{N}$ & $\mathrm{T}$ & $\mathrm{F}_{3} \mathrm{Hn}$ & $\mathrm{P}$ & $\mathrm{N}$ & $\mathrm{T}$ & FRHn & $\mathrm{P}$ & $\mathrm{N}$ & $\mathrm{T}$ & $\mathbf{R}_{1} \mathbf{T P}$ & $\mathrm{P}$ & $\mathrm{N}$ & $\mathrm{T}$ & $\mathbf{R}_{2} \mathbf{T P}$ & $\mathrm{P}$ & $\mathrm{N}$ & $\mathrm{T}$ & $\mathbf{R}_{3} \mathbf{T P}$ & $\mathrm{P}$ & $\mathrm{N}$ & $\mathrm{T}$ & RTP & $\mathrm{P} \quad \mathrm{N}$ & $\mathrm{N}$ & $\mathrm{T}$ \\
\hline $\mathrm{P}$ & 0 & 0 & 0 & $\mathrm{P}$ & 0 & 1 & 1 & $\mathrm{P}$ & 0 & & 0 & $\mathrm{P}$ & 2 & & 2 & $\mathrm{P}$ & 4 & 2 & 6 & $\mathrm{P}$ & 2 & 1 & 3 & $\mathrm{P}$ & 1 & & 1 & $\mathrm{P}$ & $\begin{array}{ll}5 & 1\end{array}$ & 1 & 6 \\
\hline $\mathrm{N}$ & 1 & 47 & 48 & $\mathrm{~N}$ & 0 & 47 & 47 & $\mathrm{~N}$ & 0 & 48 & 48 & $\mathrm{~N}$ & 28 & 18 & 46 & $\mathrm{~N}$ & 4 & 38 & 42 & $\mathrm{~N}$ & 4 & 41 & 45 & $\mathrm{~N}$ & 5 & 42 & 47 & $\mathrm{~N}$ & 93 & 33 & 42 \\
\hline $\mathrm{T}$ & 1 & 47 & 48 & T & 0 & 48 & 48 & $\mathrm{~T}$ & 0 & 48 & 48 & $T$ & 30 & 18 & 48 & $\mathrm{~T}$ & 8 & 40 & 48 & $T$ & 6 & 42 & 48 & $\mathrm{~T}$ & 6 & 42 & 48 & $\mathrm{~T}$ & 143 & 34 & 48 \\
\hline $\mathrm{P}$ & 5 & 4 & 9 & $\mathrm{P}$ & 7 & 5 & 12 & $\mathrm{P}$ & 5 & 3 & 8 & $\mathrm{P}$ & 10 & & 16 & Prot. & $\mathrm{P}$ & $\mathrm{N}$ & $\mathrm{T}$ & Prot. & $\mathrm{P}$ & $\mathrm{N}$ & $\mathrm{T}$ & Prot. & $\mathrm{P}$ & $\mathrm{N}$ & $\mathrm{T}$ & Prot. & P $\quad$ & $\mathrm{N}$ & $\mathrm{T}$ \\
\hline $\mathrm{N}$ & 4 & 35 & 39 & $\mathrm{~N}$ & 5 & 31 & 36 & $\mathrm{~N}$ & 3 & 37 & 40 & $\mathrm{~N}$ & 8 & 24 & 32 & $\mathrm{P}$ & 3 & 7 & 10 & $\mathrm{P}$ & 4 & 3 & 7 & $\mathrm{P}$ & 4 & 2 & 6 & $\mathrm{P}$ & 96 & 6 & 15 \\
\hline $\mathrm{T}$ & 9 & 39 & 48 & $\mathrm{~T}$ & 12 & 36 & 48 & $\mathrm{~T}$ & 8 & 40 & 48 & $\mathrm{~T}$ & 18 & 30 & 48 & $\mathbf{N}$ & 10 & 28 & 38 & $\mathbf{N}$ & 13 & 28 & 41 & $\mathbf{N}$ & & 35 & 42 & $\mathbf{N}$ & $15 \quad 1$ & 18 & 33 \\
\hline 3.49 & $\mathrm{CC}$ & MP & & & & nstm & 100 & ne $\mathrm{N}$ & & issada & & & & & & $\mathbf{T}$ & 13 & 35 & 48 & $\mathbf{T}$ & 17 & 31 & 48 & $\mathbf{T}$ & 11 & 37 & 48 & $\mathbf{T}$ & 242 & 24 & 48 \\
\hline Tsp & Sim & Não & $\mathrm{T}$ & $\mathrm{RR}$ & 7.33 & & & & & k & $\mathrm{R}$ & & & & & & & & & & & & & & & & & & & & \\
\hline Sim & 2 & 2 & 4 & Fishe & Exat & & & udal & & & 0,0048 & 00 & & & & & & & & & & & & & & & & & & & \\
\hline Não & 3 & 41 & 44 & & & & & audal & $\mathrm{p}$ & & 0,004 & 8643 & & & & & & & & & & & & & & & & & & & \\
\hline $\mathrm{T}$ & 5 & 43 & 48 & & & & & & & & & & & & & & & & & & & & & & & & & & & & \\
\hline
\end{tabular}

P-Positivo; N - Negativo; T - Total; Paras. - Parasitado; $\mathbf{R}_{1,2 \text { ou } 3}$ - Ritchie; $\mathbf{F}_{1,2 \text { ou } 3}$ - Faust; FR - Faust e Ritchie; RR - Risco Relativo; Tt:Trichuris trichiura;,Al: Ascaris lumbricoides; Ss: Strongyloides stercoralis; Tsp: Taenia sp; Hn:Hymenolepis nana; Ec:Entamoeba coli; En:Endolimax nana; Eh: Entamoeba hartmanni; Gl: Giardia lamblia; Paras.: Parasitado; TH: Total de Helmintos; TP: Total de Protozoários. 
coleta. Entre os parasitados o percentual de detecção foi de $16,67 \%$ (5/30) (Tabelas 3.29 à 3.32).

Em terceiro lugar, o protozoário Entamoeba hartmanni teve prevalência de $4,2 \%$, sendo identificado apenas por Ritchie (2/2) (Tabela 2.17). Nas coletas sequenciais os percentuais de detecção alcançados foram de $100,0 \%(2 / 2)$ na $1^{\mathrm{a}}$ coleta e nenhuma detecção nas outras coletas. Entre os parasitados o percentual de detecção foi de 6,67\% (2/30) (Tabelas 3.33 à 3.36).

Em quarto lugar, a única ocorrência de protozoário com potencial patogênico identificado foi zzzzzzzz $(4,17 \%)$, sendo $100,0 \%$ (2/2) identificado por Faust e $50,0 \%(1 / 2)$ por Ritchie. As medidas de efeito de Ritchie em relação à Faust podem ser visualizadas na Tabela 2.18. Nas coletas sequenciais os percentuais de detecção alcançados foram de $0,0 \%(0 / 2)$ na $1^{a}$ coleta, $50,0 \%(1 / 2)$ na $2^{\mathrm{a}}$ coleta e de $50,0 \%(1 / 2)$ na $3^{\mathrm{a}}$ coleta. Entre os parasitados o percentual de detecção foi de $6,67 \%(2 / 30)$ (Tabelas 3.37 à 3.40).

O total de protozoários detectados nas amostras sequenciais foi de $66,67 \%(10 / 15)$ na $1^{\text {a }}$ amostra, de $46,67 \%(7 / 15)$ na $2^{\mathrm{a}}$ amostra e de $40,0 \%(6 / 15)$ na $3^{\mathrm{a}}$ amostra, totalizando $50,0 \%$ (15/30) dos parasitados, com protozoários (Tabelas 3.41 à 3.44).

\section{Discussão}

\section{Análise parasitológica}

Com dificuldade para encontrar trabalhos específicos sobre enteroparasitoses em populações de catadores de material reciclável, reunimos seis trabalhos que se desdobram em oito análises parasitológicas de diferentes populações para tentar estabelecer os parasitos mais frequentes de ocorrência na população brasileira (Tabela 4), bem como estimar sua prevalência média com intervalo de confiança de 95\% e comparar com os dados de nosso trabalho.

A prevalência geral média de enteroparasitos humanos das diferentes populações apontadas na Tabela 4 foi de 48,74\% (IC 95\% - 38,94<Prevalência $<59,54$ ), variando de $10,6 \%{ }^{(12)}$ a $100,0 \%{ }^{(4)}$ Nossa prevalência média, avaliando 15 tipos diferentes de análises, foi mais baixa $(38,75 \%)$, entretanto nossa análise final de F123 e/ou R123 foi de $62,50 \%$, sendo superior à média, inclusive ultrapassando o IC 95\% da Tabela 4.

Nunes, Cunha e Marcal Júnior, ${ }^{(5)}$ através da técnica de HPJ em coletores de lixo do Município de Patrocínio, Minas Gerais (MG), encontraram que $63,64 \%$ dos indivíduos estudados, albergavam protozoários e/ou helmintos. Tanto no estudo apontado, quanto nesta pesquisa, os trabalhadores utilizavam EPI's básicos fornecidos pelas empresas, podendo esse ter sido um fator que possibilitou a similaridade dos resultados, apesar do autor ter utilizado apenas uma amostra de fezes por indivíduo para a realização do estudo.

Por outro lado, uma pesquisa desenvolvida com moradores de um bairro de periferia e catadores de um lixão, encontrou uma alta prevalência de indivíduos parasitados, sendo respectivamente, $96,86 \%$ e $100 \%$, para os grupos amostrados. ${ }^{(4)}$ Quando observamos o trabalho de Ludwig et al., ${ }^{(13)}$ sobre enteroparasitoses na população de baixo índice socioeconômico, verificamos que o total da prevalência dos indivíduos da Periferia de Cândido Mota, SP $(35,02 \%)$ é quase a metade da prevalência encontrada na presente pesquisa. Isso nos leva a acreditar que os trabalhadores das cooperativas estão mais expostos aos parasitos intestinais, podendo ser estes provenientes tanto de suas residências como dos locais de trabalho.

Cabe destacar que a demonstração microscópica de parasitos em amostras fecais nem sempre é conseguida em todas as amostras, mesmo em indivíduos altamente infectados, pois muitos cistos ou ovos são excretados de forma irregular. ${ }^{(14)}$ Fatores como volume do material examinado, número de ovos produzidos pelo parasito e carga parasitária podem influenciar no diagnóstico. Neste sentido, o número de ovos nas fezes de pacientes pode variar nas diferentes porções de um mesmo bolo fecal, esta variação estaria relacionada à espécie do parasito, à sua localização ao longo do tubo digestivo, à quantidade e ao tipo de alimento ingerido pelo hospedeiro e ao movimento peristáltico. (15) Para minimizar esta variação, recomenda-se que no mínimo três amostras colhidas em dias alternados devam ser examinadas para a presença de parasitos, principalmente para os protozoários. Este processo dispendioso aumenta a sensibilidade da análise $\mathrm{e}^{(16)}$ e a utilização combinada de vários métodos é útil, aumentando a acurácia do diagnóstico laboratorial. ${ }^{(17)}$

Um indivíduo pode estar monoparasitado ou poliparasitado (quando apresenta dois ou mais enteroparasitos). No presente estudo $62,5 \%$ estavam parasitados, dos quais $56,67 \%$ monoparasitados e $43,33 \%$ poliparasitados. Estes dados vão ao encontro de Oliveira, Silva e Costa-Cruz ${ }^{(18)}$ no qual numa área rural de Uberlândia (MG), Brasil, verificouse uma prevalência de $65,4 \%$, dos quais, $66,7 \%$ monoparasitados e $33,3 \%$ poliparasitados; e diferem de Carvalho et al. ${ }^{(19)}$ que realizaram um trabalho em 
Tabela 4 - Prevalência e comparação de técnicas na detecção de enteroparasitos na população geral e em catadores de materiais recicláveis.

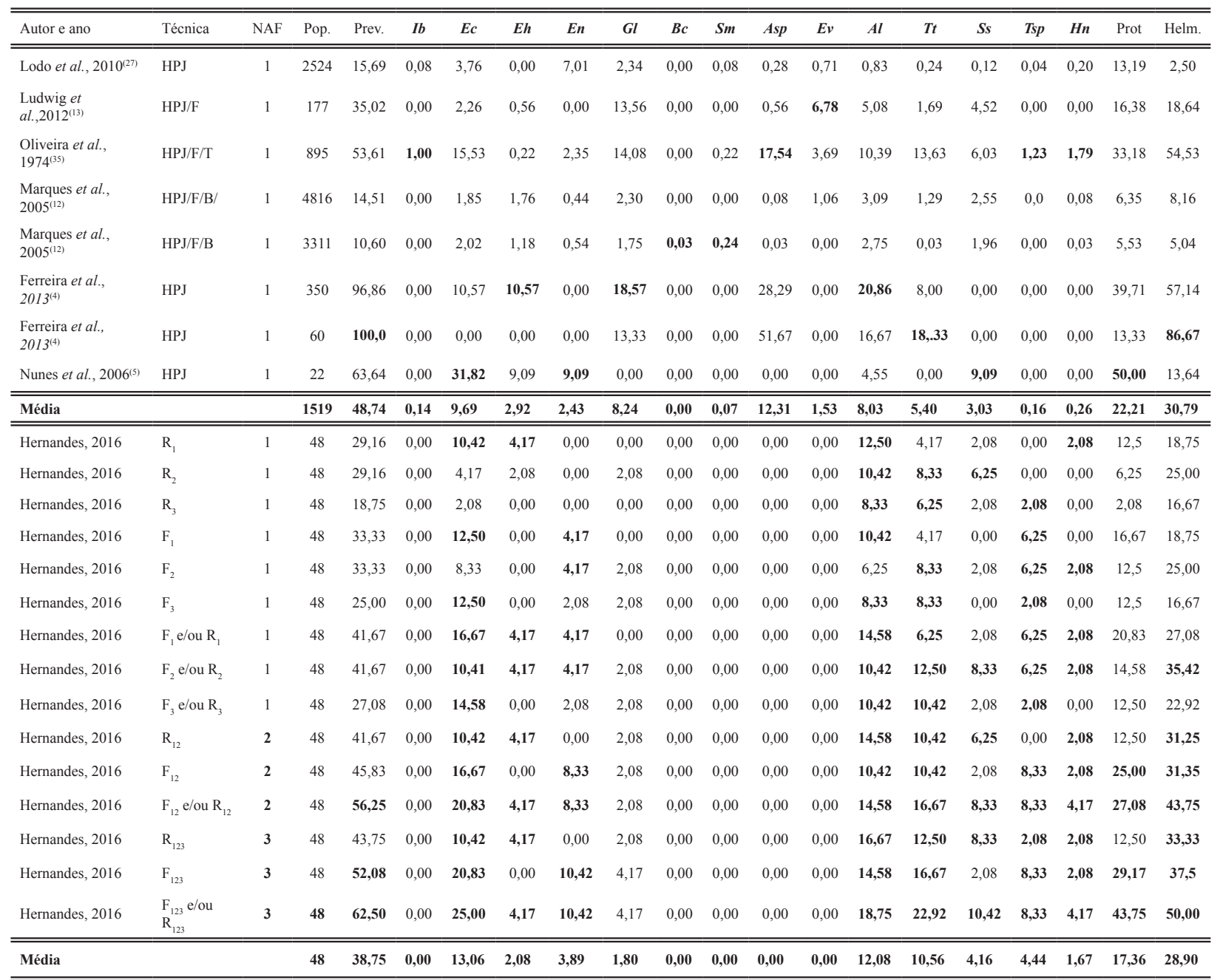

NAF - Número de amostras de fezes; HPJ - Hoffman, Pons \& Janer ; B - Baermann; F - Faust; $\mathbf{F}_{1}-$ Faust $1^{\mathrm{a}}$ amostra; $\mathbf{F}_{12}-$ Faust $1^{\mathrm{a}}$ e $2^{\mathrm{a}}$ amostras; $\mathbf{F}_{123}$ - Faust $1^{\mathrm{a}}, 2^{\mathrm{a}}$ e $3^{\mathrm{a}}$ amostras; $\mathbf{R}$ - Ritchie; $\mathbf{R}_{1}$ - Ritchie $1^{\mathrm{a}}$ amostra; $\mathbf{R}_{12}$ - Ritchie $1^{\mathrm{a}}$ e $2^{\mathrm{a}}$ amostras; $\mathbf{R}_{123}-$ Ritchie $1^{\mathrm{a}}, 2^{\mathrm{a}}$ e $3^{\mathrm{a}}$ amostras; $\mathbf{T}-$ Tamização; $\boldsymbol{I} \boldsymbol{b}$-Iodamoeba butschlii; $\boldsymbol{E} \boldsymbol{c}$ - Entamoeba coli; $\boldsymbol{E} \boldsymbol{h}$-Entamoeba hartmanni; $\boldsymbol{E} \boldsymbol{n}$ - Endolimax nana; $\boldsymbol{G l}$ - Giardia lamblia; Bc - Balantidium coli; $\boldsymbol{S m}$ - Schistosoma mansoni; Asp - Ancylostomidae spp; Ev - Enterobius vermicularis; Al -Ascaris lumbricoides; $\boldsymbol{T t}$ - Trichiuris trichiura; $\boldsymbol{S s}$ Strongyloides stercoralis; Tsp - Taenia spp.; Hn - Hymenolepis nana; Prot. - Protozoário; Helm. - Helminto. Hernandes, 2016 - dados da presente pesquisa que utilizamos para comparar com a bibliografia encontrada.

três mesorregiões de Minas Gerais, entre os escolares, e encontraram uma prevalência de $18 \%, 15.545(82 \%)$ estavam negativos, 2.863 (15\%) monoparasitados e $565(3 \%)$ poliparasitados, sendo inferiores ao nosso estudo.

A pesquisa realizada por Oliveira, Silva e CostaCruz $^{(18)}$ e nosso estudo possuem dados similares, podendo isso ser reflexo da população alvo de cada trabalho, visto que os trabalhadores de materiais recicláveis moram nas vilas e bairros da cidade estudada, local similar, em termos de saneamento, fator econômico e ambiental que os moradores da área rural da pesquisa mencionada; além disso, analisaram mais de uma amostra por indivíduo e realizaram duas técnicas parasitológicas, o que aumenta a probabilidade de achados parasitológicos. Já com relação aos escolares ${ }^{(19)}$ os dados encontrados podem ter sido subestimados, visto a técnica parasitológica utilizada (não utilizou nenhuma outra para comparar os achados) e o número de amostras coletadas (não relata no artigo quantas amostras por indivíduo foram analisadas, podendo ter sido insuficiente para retratar o estado real da população estudada). 
Com relação aos protozoários diagnosticados, apenas G. lamblia possui caráter patogênico. No entanto, o diagnóstico de parasitos comensais é importante, pois eles têm o mesmo mecanismo de contaminação dos patogênicos e podem servir como um bom indicador de contaminação e, portanto, das condições sociossanitárias. ${ }^{(5)}$

Quando observamos as análises da Tabela 4, E. coli foi o protozoário mais prevalente, sendo este considerado um protozoário comensal, com uma média de $9,69 \%$ variando de $0,0 \%$ no estudo de Ferreira et al., ${ }^{(4)}$ a $31,82 \%$ no de Nunes; Cunha; Marçal Júnior. (5) Nesta pesquisa, também se encontrou uma maior prevalência deste protozoário comensal $(25,00 \%)$, sendo este valor encontrado na análise realizada com as três amostras e as duas técnicas em paralelo.

$\mathrm{Na}$ Tabela 4 as prevalências para G. lamblia, variaram de $0,0 \%$ a $18,57 \%$ com uma média de $8,24 \%$. Nesta pesquisa, a prevalência foi $4,17 \%$, concordando com Sogayar e Guimarães, ${ }^{(20)}$ onde relatam que no Brasil, a prevalência varia de $4 \%$ a $30 \%$. Este protozoário foi encontrado uma vez em poliparasitismo com E. coli e E. hartmanni e outra vez em poliparasitismo com T. trichiura e E. coli. Faust foi mais sensível na detecção, concordando com Bica, Dillenburg e Tasca ${ }^{(1)}$ que avaliando a performance de Faust e HPJ, encontraram maior sensibilidade para Faust, o que também está de acordo com escassos relatos da literatura, como os trabalhos de Amato Neto et al. ${ }^{(21)}$ e Gates e Nolan. ${ }^{(22)}$

A transmissão da giardíase ocorre através da água, do consumo de vegetais, legumes e frutas contaminadas pelos cistos, de manipuladores de alimentos, do contato direto (fecal-oral), principalmente em asilos, creches, clínicas psiquiátricas. Considera-se, ainda, a transmissão por meio de artrópodes, como as moscas e baratas, através de seus dejetos ou regurgitação.(23)

Já com relação aos helmintos, $T$. trichiura foi o parasito mais diagnosticado na presente pesquisa $(22,92 \%)$, variou de $0,0 \%{ }^{(5)}$ a $18,33 \%^{(4)}$ (Tabela 4 ). Essa diferença pode ter acontecido pela utilização de mais uma técnica, visto que os trabalhos mencionados acima utilizaram apenas a técnica de HPJ, sendo esta de sedimentação espontânea. Este parasito é transmitido pelo solo, sendo predominante em regiões tropicais e subtropicais do mundo, com falta de saneamento. (24) A infecção é adquirida pela ingestão de água ou alimentos contaminados e é geralmente assintomática. No entanto, quando ela progride de uma infecção leve a grave, sinais clínicos específicos se põem de manifesto, ${ }^{(25)}$ como a insurgência da chamada Síndrome Disentérica por Trichuris (SDT), especialmente em crianças, sendo caracterizada por diarreia mucóide, sangramento e prolapso retal complicado com infecção secundária bacteriana grave. ${ }^{(26)}$

A prevalência geral da Tabela 4 , quanto à presença de $A$. lumbricoides, variou de $0,83 \%{ }^{(27)}$ a $20,86 \%,{ }^{(4)}$ com uma prevalência média de $8,03 \%$. Nossa prevalência foi superior, coincidindo com o trabalho de Ferreira et al., ${ }^{(4)}$ onde um dos grupos pesquisados foram os catadores, no entanto estes se encontravam em um lixão. Estas prevalências mostram que mesmo os catadores das cooperativas de triagem utilizando EPI's e triando resíduos segregados pela população, ainda assim encontram-se parasitados.

A ascaridíase é a helmintíase mais difundida mundialmente. $^{(28)}$ Fatores como genética, nível socioeconômico, exposição ambiental e resposta imune, são fatores determinantes para a infecção. Pessoas com esta parasitose podem apresentar colecistite, colangite, pancreatite, apendicite, necrose intestinal. Complicações podem ocorrer em todas as idades, mas crianças entre 3 e 5 anos são mais suscetíveis a obstrução intestinal por $A$. lumbricoides. ${ }^{(6)}$

Com relação ao helminto $S$. stercoralis, visualizase na Tabela 4 que a média de prevalência foi de $3,03 \%$, enquanto que no presente estudo obteve-se uma prevalência de 10,42\%. Infecções por Strongyloides spp. ocorrem em uma variedade de espécies de mamíferos em todo o mundo, particularmente nas regiões tropicais e temperadas com clima mais quente que favorecem a sobrevivência de estágios de desenvolvimento do parasito no solo. Diferentes espécies variam na sua especificidade de hospedeiro, a espécie $S$. stercoralis é encontrada em seres humanos, primatas e animais de companhia como cães e gatos, portanto, consideradas zoonóticas. ${ }^{(29)}$ Problema este caracterizado no presente estudo, onde na cooperativa em que foram diagnosticados catadores com S. stercoralis, notou-se a presença de cães e gatos tanto dentro das cooperativas quanto no entorno.

Duas espécies de Taenia são frequentes parasitos intestinais humanos. T. saginata é a mais frequente e é encontrada na maioria dos países onde a carne é consumida. T. solium é endêmica na América Latina, África e alguns países asiáticos. A infecção ocorre quando humanos ingerem carne de bovinos ou de suínos contaminadas. ${ }^{(30)}$ No teste parasitológico não é possível distinguir qual espécie de Taenia spp. está sendo diagnosticada. No presente trabalho a prevalência foi 
superior aos estudos da Tabela 4. Isto pode ter ocorrido pelo consumo de carne mal passada e contaminada, ${ }^{(31)}$ uma vez que foi encontrado uma correlação positiva em dois indivíduos parasitados com Taenia sp. e que tinham o hábito de consumir carne mal passada (Tabela 3.49).

Hymenolepis nana está associada à baixa imunidade e falta de higiene, ocorrendo em pessoas com alto grau de promiscuidade. A pessoa também pode carregar os ovos deste parasito embaixo das unhas. No Brasil, este helminto apresenta maior incidência na região Sul devido à existência de ambientes fechados em função do frio e ambientes coletivos como creches. ${ }^{(32)}$ Este parasito pode realizar ciclo heteroxênico, onde os insetos (pulgas de ratos e cães, e carunchos de cereais - Tenebrio molitor) fazem o papel de hospedeiros intermediários. ${ }^{(33)}$

Na presente pesquisa a prevalência de catadores com $H$. nana foi de $4,17 \%$, ao passo que a prevalência geral mostrada na Tabela 4 foi $0,26 \%$. Isto pode ter ocorrido tanto pela transmissão fecal-oral (papel higiênico, fraldas descartáveis no lixo) como pela interferência de insetos. Segundo Lopes et al. ${ }^{(34)}$ no Brasil, assim como em outros países em desenvolvimento, as helmintíases constituem enorme problema de saúde pública, por sua morbidade e extrema dificuldade de terem as suspeitas diagnosticadas e controladas.

\section{Conclusão}

As técnicas parasitológicas testadas no estudo são de concentração de amostra, sendo estas mais indicadas que aquelas onde ocorre a sedimentação espontânea ou exame direto a fresco. Diante de todas as análises realizadas, verificou-se que as técnicas de Faust e Ritchie mostraram-se complementares, ou seja, dependendo do tipo de parasito a ser detectado, a eficiência de alguma é maior ou menor. Entretanto recomenda-se utilizar as duas em combinado para aumentar a sensibilidade e especificidade das mesmas.

Com relação aos dados parasitológicos do presente estudo, pode-se perceber que a prevalência de enteroparasitoses na população de catadores foi superior à população geral de baixa renda.

\section{Referências}

1 Bica VC, Dillenburg AF, Tasca T. Diagnóstico laboratorial da giardiose humana: comparação entre as técnicas de sedimentação espontânea em água e de centrífugo-flutuação em solução de sulfato de zinco. Rev HCPA. 2011;31(1):39-45.

2 Sant'Anna LML, Oliveira FJ, Melo CM. Estudo comparativo de técnicas parasitológicas baseada no princípio de sedimentação espontânea (Hoffman) e Parasitokit $^{\circledR}$. Scire Salutis. 2013;3(1):6-15. Doi: 10.6008/ESS2236-9600.2013.001.0001

3 Alves, APSM, Coelho MDG, Santos IA, Bozo LSO, Maciel LTR. Contaminação em logradouros do Município de Pindamonhangaba-SP, por parasitos potencialmente zoonóticos em fezes caninas. Rev Ciên Saúde. 2016;1(1):45-50.

4 Ferreira VS, Lima AGD, Pessoa CS, Paz FSS, Jesus J. Estudo comparativo das enteroparasitoses ocorrentes em duas áreas de Barreiras, Bahia. Natureza on line. 2013;11(2):90-95.

5 Nunes ALBP, Cunha AMO, Marçal Júnior O. Coletores de lixo e enteroparasitoses: o papel das representações sociais em suas atitudes preventivas. Ciênc Educ. 2006;12(1):25-38.

6 AndradeAM,PerezY,LopezC, Collazos SS, Andrade $\mathrm{AM}$, Ramirez GO, et al. Intestinal obstruction in a 3-Year-Old Girl by Ascaris lumbricoides infestation: case report and review of the literature. Medicine (Baltimore). 2015 Apr;94(16):e655. doi: 10.1097/MD.0000000000000655.

7 Gutberlet J, Baeder AM, Pontuschka NN, Felipone SMN, Santos TLF. Participatory research revealing the work and occupational health hazards of cooperative recyclers in Brazil. Int J Environ Rev Public Health. 2013;10:4607-4627.

8 Lenis V, López YL, Cuadros YM. Health and informal work conditions among recyclers in the rural area of Mendellin, Colombia Rev Saude Publica. 2012 Oct;46(5):866-74. 
9 Dall'Agnol CM, Fernandes FS. Saúde e auto cuidado entre catadores de lixo: vivências no trabalho em uma cooperativa de lixo reciclável. Rev. Latino-Am. Enfermagem. 2007; 15(spe): 729735.

10 Faust EC, D’Antoni JSL, Odom V, Miller MJ, Peres C, Sawitz W. A critical study of clinical laboratory technics for the diagnosis of protozoan cysts and helminth eggs in feces. Am J Trop Med Hyg. 1938;18(2):169-183.

11 Hoffmann RP. Diagnóstico de parasitismo veterinário. Porto Alegre: Sulina; 1987.

12 Marques SMT, Bandeira C, Quadros RM. Prevalência de enteroparasitoses em Concórdia, Santa Catarina, Brasil. Parasitol Latinoam, 2005;60:78-81.

13 Ludwig KM, Ribeiro ALT, Conte AOC, Decleva DV, Ribeiro JTD. Ocorrência de enteroparasitoses na população de um bairro da cidade de Cândido Mota-SP. J Health Sci Inst. 2012;30(3):271-276.

14 American Society of Parasitologists. Subcommittee on laboratory standards: procedures suggested for use in examination of clinical specimens for parasitic infection. J. Parasitol. 1997;63:959-960.

15 Araújo AJUS, Kanamura HY, Dias LCS, Gomes JF, Araújo SM. Coprotest ${ }^{\circledR}$ quantitativo: quantificação de ovos de helmintos em amostras fecais utilizandose sistema de diagnóstico comercial. J. Bras. Patol. Med. Lab. 2003 June;39(2):115-124.

16 Marti H, Koella JC. Multiple stool examinations for ova and parasites and rate of false-negative results. J Clin Microbiol. 1993 Nov; 31(11):30445.

17 Menezes RAO, Gomes MSM, Barbosa FHF, Machado RLD, Andrade RF, D'Almeida Couto, AAR. Sensibilidade de Métodos Parasitológicos Para o Diagnóstico das Enteroparasitoses em Macapá - Amapá, Brasil. BioTerra. 2013;13(2):6673.
18 Oliveira MC, Silva CV, Costa-Cruz JM. Intestinal parasites and commensals among individuals from a landless camping in the rural area of Uberlandia, Minas Gerais, Brazil. Rev. Inst. Med. Trop. 2003;45(3):173-176. Doi:10.1590/S003646652003000300011 .

19 Carvalho OS, Guerra HL, Campos YR, Caldeira RL, Massara CL. Prevalência de helmintos intestinais em três mesorregiões do Estado de Minas Gerais. Rev. Soc. Bras. Med. Trop. 2002;35(6):597-600.

20 Sogayar MITL, Guimarães S. Giardia. Em: Neves DP, Melo AL, Linardi PM, Vitor RWV. Parasitologia humana. $11^{\text {th }}$ ed. São Paulo: Atheneu; 2005. p. 121-126.

21 Amato Neto V, Campos R, Pinto PL, Matsubara L, Braz LM, Miyamoto A. Evaluation of the usefulness of the Coprotest for parasitologic examination of feces. Rev Hosp Clin Fac Med. 1989 Jul-Aug;44(4):153-5.

22 Gates MC, Nolan TJ. Comparison of passive fecal flotation run by veterinary students to zincsulfate centrifugation flotation run in a diagnostic parasitology laboratory. J Parasitol. 2009;95(5):1213-4

23 Pereira MGC, Atwill ER, Barbosa AP. Prevalence and associated risk factors for Giardia lamblia infection among children hospitalized for diarrhea in Goiânia, Goiás state, Brazil. Rev. Inst. Med. Trop. 2007;49(3):139-145.

24 Azira NMS, Zeehaida M. Severe chronic iron deficiency anemia secondary to Trichuris dysentery syndrome - a case report. Trop Biomed. 2012 Dec;29(4):626-31.

25 Bianucci R, Torres EJL, Santiago JMFD, Ferreira LF, Nerlich AG, Souza SMM. Trichuris trichiura in a post-Colonial Brazilian mummy. Mem Inst Oswaldo Cruz. 2015;110(1):145-147.

26 Cooper ES, Whyte-Alleng CAM, Finzi-Smith JS, McDonald TT. Intestinal nematode infections in children: the patho-phisyological price paid. Parasitology. 1992;104 Suppl:S91-103. 
27 Lodo M, Oliveira CGB, Fonseca ALA, Caputto LZ, Packer MLT, Valenti VE, et al. Prevalência de enteroparasitas em município do interior paulista. Rev. Bras. Crescimento Desenvolv. Hum. 2010;20(3):769-777.

28 Macedo LMC, Rey L. Enteroparasitoses em gestantes e puérperas no Rio de Janeiro. Cad. Saúde Pública. 1996;12(2):383-388.

29 Viney ME, Lok JB. The biology of Strongyloides spp. In: The C. Elegans Research Community; WormBook WormBook: the online review of c. elegans biology [Internet]. 2007. [cited 2016 July 20]. Disponível em: https://www.ncbi.nlm.nih.gov/ books/NBK19795/

30 Gonçalves MLC, Araújo A, Ferreira LF. Human intestinal parasites in the past: new findings and a review. Mem Inst Oswaldo Cruz. 2003;98(Suppl. I):103-118.

31 Sindicato da Indústria de Carnes e Derivados no Estado do Rio Grande do Sul. Uruguai passa a Argentina em consumo de carnes. [Internet]. 2010. [citado 2016 dez 15]. Disponível em: http:// revistasaboresdosul.com.br/informações/ver. php?id=24529=Consumo_de_carne.

32 Ferreira MU. Parasitologia contemporânea. Rio de Janeiro: Guanabara Koogan; 2012.

33 Neves DP. Parasitologia humana. 12. ed. São Paulo: Atheneu; 2011.

34 Lopes AJ, Rocha JL, Kirk KM, Jansen JM. Síndrome de Löffler. Pulmão. 2005;14(4):325-327.

35 Oliveira MR, Barbosa MA, Salata E, Sogayar MITL, Sogayar R, Corrêa FMA. Prevalência de enteroparasitas na população urbana do $2^{\circ}$ subdistrito de Botocatu. Rev. Saúde Públ. $1974 ; 8(2): 213-234$. 